\title{
Enabling and Emerging Sensing Technologies for Crowd Management in Public Transportation Systems: A Review
}

This paper was downloaded from TechRxiv (https://www.techrxiv.org).

\section{LICENSE}

CC BY 4.0

SUBMISSION DATE / POSTED DATE

$14-10-2021 / 10-12-2021$

\section{CITATION}

Darsena, Donatella; Gelli, Giacinto; ludice, Ivan; Verde, Francesco (2021): Enabling and Emerging Sensing Technologies for Crowd Management in Public Transportation Systems: A Review. TechRxiv. Preprint. https://doi.org/10.36227/techrxiv.16811191.v2

$\mathrm{DOI}$

10.36227/techrxiv.16811191.v2 


\title{
Enabling and Emerging Sensing Technologies for Crowd Management in Public Transportation Systems: A Review
}

\author{
Donatella Darsena, Senior Member, IEEE, Giacinto Gelli, Senior Member, IEEE, Ivan ludice, \\ and Francesco Verde, Senior Member, IEEE
}

\begin{abstract}
Management of crowd information in public transportation (PT) systems is crucial to foster sustainable mobility, by increasing the user's comfort and satisfaction during normal operation, as well as to cope with emergency situations, such as pandemic crises, as recently experienced with COVID-19 limitations. This paper presents a taxonomy and review of sensing technologies based on Internet of Things (IOT) for real-time crowd analysis, which can be adopted in various segments of the PT system (buses/trams/trains, railway/subway stations, and bus stops). To discuss such technologies in a clear systematic perspective, we introduce a reference architecture for crowd management, which employs modern information and communication technologies (ICT) in order to: (i) monitor and predict crowding events; (ii) adapt in real-time PT system operations, by modifying service frequency, timetables, routes, and so on; (iii) inform in realtime the users of the crowding status of the PT system, by means of electronic displays installed inside vehicles or at bus stops/stations, and/or by mobile transport applications. It is envisioned that the innovative crowd management functionalities enabled by ICT/IOT sensing technologies can be incrementally implemented as an add-on to traditional intelligent transportation system (ITS) platforms, which are already in use by major PT companies operating in urban areas. Moreover, it is argued that, in this new framework, additional services can be delivered, such as, e.g., on-line ticketing, vehicle access control and reservation in severely crowded situations, and evolved crowd-aware route planning.

Index Terms-COVID-19, crowd management, intelligent transportation system (ITS), Internet of Things (IoT), public transportation (PT) systems, sensing technologies, sustainable mobility, smart cities.
\end{abstract}

\section{INTRODUCTION}

$\mathbf{S}$ INCE 2002, the European Commission has promoted across Europe and beyond a campaign supporting the use of public transportation (PT) systems as "a safe, efficient, affordable, and low-emission mobility solution for everyone" [1]. To cope particularly with PT limitations in urban areas, a key role is expected to be played by intelligent transportation systems (ITSs) [2], which leverage information and communication technologies (ICT) to enable automated collection of transportation data, used to make transport safer, more efficient, more reliable, and more sustainable.

Manuscript received December 1, 2021; revised xx yy, 2021; accepted $x x y y, 2021$. The editor coordinating the review of this manuscript and approving it for publication was XXX.

D. Darsena is with the Department of Engineering, Parthenope University, Naples I-80143, Italy (e-mail: darsena@uniparthenope.it). I. Iudice is with Italian Aerospace Research Centre (CIRA), Capua I81043, Italy (e-mail: i.iudice@cira.it). G. Gelli and F. Verde are both with the Department of Electrical Engineering and Information Technology, University Federico II, Naples I-80125, Italy [e-mail: (f.verde, gelli)@unina.it].

D. Darsena, G. Gelli, and F. Verde are also with National InterUniversity Consortium for Telecommunications (CNIT).
Two different classes of spatio-temporal data are available in state-of-the-art ITSs for PT: (i) vehicle data, such as location and speed, which are obtained (usually in real-time) by means of automated vehicle location (AVL) [3] systems, based on satellite localization techniques; and (ii) passenger data, such as the number of passengers boarding a bus/train or entering a station, which can be obtained by means of automatic passenger counting (APC) systems [4] or automatic fare collection (AFC) systems [5]. Such data are commonly used as inputs for optimization and planning strategies for PT systems, which are surveyed in [6] at four different levels: strategic, tactical, operational, and real-time. In particular, it is observed in [6] that lack of passenger arrival information, especially in realtime, is a limiting factor for accurate studies. In many ITSs, indeed, real-time location data are available only for vehicles, which are used both to provide trip information to passengers and for medium-to-long term management and monitoring of the service.

With the advent of Internet of Things (IoT) technologies, fine-grained and real-time passenger data collection is becoming a feasible task, especially in smart cities [7]. Pervasive use of mobile and portable devices, equipped with different 
sensors, allows one to gather huge quantities of data in urban scenarios $[8,9,10]$, which can be used for different applications and tasks [11]. In particular, the fifth generation (5G) of cellular networks is potentially able to support massive IoT connections [12], where billions of smart devices can be connected to the Internet, and can be easily located and tracked; these features will be further extended by forthcoming sixth generation (6G) networks [13].

Recently, some researchers have argued that the quality of service $(\mathrm{QOS})$ perceived by PT users, as well as their travel satisfaction/quality of experience (QoE), are seriously affected by crowding $[14,15,16]$. To cope with this issue, it is required to acquire real-time reliable and capillary information about the crowding status of PT rail or road vehicles (e.g., buses, trams, and trains) and the related access infrastructures (e.g., bus stops and metro/railway stations). Indeed, it is stated in [17] that "the availability of real-time passenger demand data can significantly improve the performance of control models in case of overcrowding".

Motivated by the previous needs, new modeling, planning and management strategies that collect real-time crowding data and use it to improve QoS/QoE in PT systems are appearing in the literature $[18,19,20,21,16]$, and will be referred in the following as crowd management [22]. Crowd management systems are composed by crowd monitoring/analysis and crow control components [23]: the former includes a network of physical sensors aimed at detecting crowds and estimating their parameters, whereas the latter includes prediction, decision making and control strategies aimed at managing the crowd events.

The problem of crowd management in PT systems has emerged dramatically during coronavirus disease (COVID-19) pandemic, which have first spurred our interest to this topic (see Section II for a discussion). During the acute outbreak phase, overcrowding of buses and trains needed to be strictly avoided to protect people from contagion, which required emergency measures, such as, e.g., limiting to $50 \%$ the service capacity. These measures are generally not sustainable in the long-term, since they shift a significant portion of passengers to private transportation. Moreover, the problem of overcrowding in PT systems must be tackled in a smarter and more structural way, since many experts predict that, in the future, recurrent waves of pandemic outbreaks could become the norm rather than the exception.

Although state-of-the-art AVL/APC/AFC systems collect a large amount of data about vehicles and passengers in ITS systems, often they are not useful to achieve real-time crowd monitoring and control. However, many ICT/IoT sensing technologies for crowd monitoring are already available or will be available in smart cities [7]. A recent special issue [24] reports the cutting-edge advances and ICT technologies pertaining to the seamless integration of sensors with the transportation infrastructure. The main focus of the papers in [24] is on sensing technologies for private transportation systems, oriented to autonomous driving [25, 26], intelligent fault detection [27, 28], electric charging optimization [29], road condition monitoring [30,31], precise fleet management [32], speed detection and accident avoidance [33, 34]: in the same issue, less attention is dedicated to the integration of sensing technologies within PT systems. Moreover, sensing techniques for crowd monitoring are not specifically discussed.

In this paper, we argue that although many sensing technologies for crowd monitoring are already available, the diffusion of crowd management techniques in modern PT systems is hindered by the lack of a structured framework and reference architecture. Motivated by this observation, we pursue two main goals in this paper: (i) to present a survey and taxonomy of crowd monitoring technologies for PT systems based on ICT/IoT technologies; (ii) to discuss their adoption into a reference framework, aimed at integrating the legacy ITS (already available in many PT systems in urban areas) with the new crowd management functionalities. Although some reviews regarding techniques for crowd monitoring inside PT vehicles exist (see $[4,35]$ ), to the best of our knowledge, this is the first review addressing sensing technologies for crowd management in all the different segments of the PT systems.

In our vision, the crowd management functionalities are built upon a distributed IoT subsystem, composed by a capillary network of heterogenous active/passive sensors, aimed at monitoring passenger crowding in buses, trams, and trains, at bus stops, and in railway/metro stations. By means of a communication infrastructure, the acquired measures are transmitted in real-time to a smart subsystem, which performs crowd control functionalities. Crowding information can also be reported (in aggregated form, for privacy concerns) to PT users, by means of displays installed inside vehicles or at stations/bus stops, or through mobile transport apps (e.g., Moovit or proprietary operator applications).

Real-time knowledge of crowding data can be used by PT operators for fast or even proactive adaptation of some service features (e.g., increasing the service frequency, reallocating vehicles from one line to another, planning alternative routes), in order to cope with spatially and/or temporally localized crowding situations, which cannot be tackled by conventional (statistical) tools used in transportation system design, such as, e.g., origin-destination (O-D) flow analysis. The new crowd management functionalities essentially achieve two scopes: (i) to improve QoS/QoE of passengers, thereby fostering PT system usage; (ii) to allow for safe PT usage during exceptional events like a pandemic outbreak, such as COVID19.

\section{A. Paper organization}

Section II highlights the adverse impact of COVID-19 pandemic outbreak on sustainable mobility and PT systems. In Section III, the main aspects of crowd management in PT systems are discussed and a crowd management reference architecture is introduced. A taxonomy and review of various sensing solutions for crowd management and their application in different PT scenarios is discussed in Section IV. Innovations and advantages provided by the adoption of the new crowd management functionalities are highlighted in Section V. Finally, conclusions are drawn in Section VI. 


\section{SustainABLE MOBILITY IN THE COVID-19 ERA}

During the last two years, the transport sector and mobility - in particular PT systems in urban areas - have been seriously affected by COVID-19 pandemic. A survey carried out in China in 2020 [36] estimated that, as a consequence of the outbreak, the use of private cars will be roughly doubled, increasing from $34 \%$ to $66 \%$, whereas the use of public transports (buses/metros) will be more than halved, dropping down from $56 \%$ to $24 \%$. Furthermore, due to the lack of trust in PT systems, more than $70 \%$ of the surveyed people not owning a car declared their intention to buy a new one, with negative consequences on the environment (landscape and air pollution) in urban areas. Other recent studies (see, e.g., [37, 38]) have highlighted that COVID-19 pandemic has seriously discouraged the use of PT systems.

To counteract the shift to private car usage during COVID19 pandemic, national governments have implemented different strategies. A widespread measure [39] has been to favor the use of individual sustainable mobility and micromobility means, such as bikes, electrical scooters, and segways, by deploying the related infrastructures (emergency bike lanes) or empowering vehicle sharing services, which can shift to this transport mode a certain percentage of short and mediumdistance trips.

However, owing to the large number of passengers carried by PT systems in urban areas, it is of utmost importance to adopt measures aimed at safe and reliable PT usage in such a scenario. During the acute phase of the outbreak, severe anti-COVID-19 measures were adopted [40] aimed at minimizing the contagion risk, such as back-door boarding, cashless operations, frequent sanitization of vehicles and stations, enforcing social distances, limiting the service capacity, and requiring the passengers to wear face masks. Other antiCOVID-19 measures were applied to PT system operations, such as modifying timetables, frequencies, paths, leveraging modal integration, and so on. Some of these measures, like increasing PT service frequency or introducing extraordinary trips to compensate for the reduced vehicle capacity, are seen by PT companies as effective, but not sustainable in the long term, due to the limited number of drivers and vehicles [41].

Generally speaking, the COVID-19 pandemic has pushed towards a critical rethinking of many economical, social, and cultural habits, not only those related to sustainable mobility. A plethora of innovative solutions have been proposed to cope with this new challenge, many of them employing ICT/IoT technologies. In [42], the use of new technologies, such as IoT, unmanned aerial vehicles (UAVs), artificial intelligence (AI), blockchain and 5G, has been considered for managing the impact of COVID-19 in health applications. In [43, 44] a review of technologies for social distancing has been provided, with emphasis on wireless technologies for positioning, including crowd detection and people density estimation.

As far as sustainable mobility is concerned, a review of the PT planning literature can be found in [45] from the perspective of the changes in demand patterns and limited capacity requirements associated with the COVID-19 pandemic crisis. It is evidenced that, besides reducing the service capacity to adhere to physical distancing measures, PT service providers worldwide have resorted to limiting service span in order to reduce operational costs as a consequence of the reduction in catchment area, by canceling certain services or closing some stations. In [45] it is pointed out the importance to develop and deploy methods that are able to maintain the functionality of PT systems, while minimizing the public health risks; it is suggested that some changes in service provision can be made at the tactical planning phase, by modifying timetables and/or service frequencies.

Some recent studies have explored the possibility to rely on ICT to organize and facilitate human mobility during the pandemic. In [46], the Authors propose to use a machinelearning (ML) based approach to trace daily train travelers in different age cohorts of $16-59$ years (i.e., the less vulnerable age-group) and over 60 (i.e., the more vulnerable age-group) in order to recommend certain times and routes for safe traveling. In this work, many ICT technologies, such as WiFi, Radio-Frequency IDentification (RFID), Bluetooth, and Ultra WideBand (UWB), are employed. Using a dataset of the London underground and overground network, different ML algorithms are compared in [46] to properly classify different age group travelers, showing that the Support Vector Machine (SVM) approach performs better to predict the mobility of travelers and achieves high accuracy (more than $80 \%$ ).

In [47], a comprehensive review on human mobility research using big data is carried out: big data collected thanks to the pervasive use of ICT/IoT can help, indeed, discover the relationships between human mobility and resource use, thus entailing great opportunities for smart city development. In [48], instead, the Authors pursue the objective of identifying data sources and ML approaches to properly estimate the impact of COVID-19 on human mobility reduction. In particular, [48] investigates the consequences of the pandemic on mobility patterns of urban populations, by quantifying even the impact of mobility reduction on improving air quality in urban areas.

\section{Crowd management in PT Systems}

A general introduction to crowd management in transportation systems is provided in [22], where some limitations of state-of-the-art ITSs are highlighted, and the potentials of the new approach are discussed, together with a brief introduction to crowd monitoring/analysis techniques. A simulation-based approach, which assesses the benefits of real-time crowding information (RTCI) dissemination on passenger travel choices, is introduced and discussed in [16, 49]. In [49] it is shown that providing RTCI at bus stops might help reduce the deleterious "bus bunching" effect. In [16] a complete framework for RTCI modeling in PT systems is introduced, which incorporates RTCI in a dynamic path choice model: the new methodology is tested on a simplified model of the urban PT system of Kraków, Poland, showing that RTCI dissemination contributes to a more efficient distribution of passenger loads in the PT network, improving travel confort and reducing waiting time. In [15] different aspects of passenger crowding in PT systems are discussed, related to demand, supply, and operations, 
including effects on route and bus choice and passengers' wellbeing.

A field application of crowd management strategies to PT systems is considered in [18, 20]. The proposed solution, tested in the municipal bus infrastructure of Madrid, Spain, estimates the current number of passengers in a bus by exploiting the properties of the existing WiFi connections (see Section IV-H) and incorporates such information in a bus navigation system, which is capable of giving crowd-aware route recommendations.

An essential component of crowd management is crowd prediction. Prediction approaches can be classified, on the basis of the prediction methodology, into model-based methods or data-driven ones. Model-based methods include timeseries analysis, regression modeling, hidden Markov models and Kalman filtering models. Due to the highly nonlinear and random nature of crowds, data-driven approaches have recently gained significant attention, including $k$-nearest neighbor ( $k$ NN) methods, artificial neural networks techniques, ML and deep-learning (DL) techniques.

Although crowd/traffic prediction and mobility forecasting are considered in several papers, their application to PT systems is a relatively new topic: some recent studies are [50, $51,52,53,54]$. In [52], a predictive model for bus crowding is proposed and tested on a dataset taken from the Pittsburgharea bus using ML techniques. In [53] a data-driven approach is considered to perform car-specific metro train crowding prediction, aimed at providing in-vehicle or station crowding information. Real-time crowd estimation in [53] is based on load sensors (see Section IV-B) and is used together with historical data to perform crowd prediction, which is tested with data gathered on a metro line in Stockholm, Sweden. In [54] a framework for personalized (i.e., user-specific) crowd prediction is proposed, which takes into account not only loading data, but also other parameters that affect user confort, such as, e.g., seat availability.

\section{A. A reference architecture for crowd management}

From the previous discussion, it is apparent that integration of ICT/IoT sensing technologies into PT systems for crowd management is fragmentary and their potentials are not fully exploited to date. To bring together these technologies in a systematic and common scenario, we introduce a crowd management reference architecture for ITS, whose scope is to integrate/augment the ITS system already available in a urban PT system with the new crowd management functionalities, aimed at smart and proactive control and reduction of passenger crowding.

The key idea is to integrate heterogenous sensing and communication technologies, in dependence on the operation scenario and the ICT infrastructure available in the urban area where the system must be implemented. To achieve such a goal in practice, strong interdisciplinary design skills are needed, including transportation engineering, telecommunications, computer science, electronics, data analysis, and AI.

In our vision, the reference architecture encompasses (Fig. 1) three subsystems:

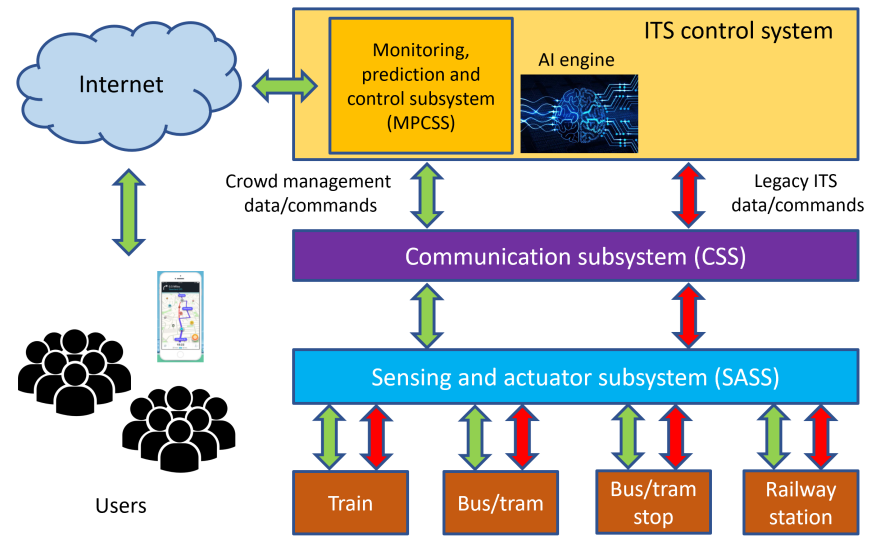

Fig. 1. A crowd management reference architecture for ITS.

1) the sensing and actuator subsystem (SASS);

2) the communication subsystem (CSS);

3) the monitoring, prediction and control subsystem (MPCSS).

The architecture involves new data flows (marked as green arrows in Fig. 1) for crowd management, in addition to existing data exchanges (red arrows) commonly used in state-of-theart ITS systems for PT services. The core and most innovative part of the system is the SASS, with particular reference to sensing technologies, whereas the actuator component mainly encompasses the flow of information toward the users, carried out by audio speakers, variable-message panels, or displays, which are typically already present in the PT system, or can be readily installed at the bus stops, as well as in railway stations, and also inside vehicles. In Section IV, we provide a taxonomy and discussion of the different sensing technologies than can be utilized in the SASS.

As regards the CSS, its characteristics are strongly dependent on the communications infrastructure available in the urban area. In general, this might encompass public wireless networks (such as cellular networks) and/or private wired and/or wireless networks owned by the operator, such as, e.g., Global System for Mobile Railway (GSM-R) or LTE for Railway (LTE-R). To cope with this heterogeneity, it is envisioned that, at the protocol level stack, the CSS can be readily interfaced with the other subsystems by means of standard or open interfaces and/or using simple adaptation layers.

The MPCSS performs data collection and real-time crowd prediction, possibly employing $\mathrm{AI}$ and ML/DL techniques. Based on such predictions, modifications to the transport services can be implemented in real-time, the related control data are sent to service operators (drivers, supervisors, etc.), whereas service information is sent to the passengers by means of displays and/or mobile transport apps. This information could be notified by the same applications to all the users of the PT, so as to discourage the access to overcrowded stations and/or bus stops, and propose alternative travel solutions. The MPCSS can be strongly integrated (and typically colocated) with the ITS control system of the PT service operator. 


\section{Sensing teCHNOLOGIES FOR CROWd MANAGEMENT}

From the transportation perspective, our taxonomy of sensing technologies for PT systems considers (see also Fig. 1) the following scenarios:

1) train and bus/tram vehicles (indoor scenarios);

2) railway/metro stations (indoor/outdoor scenarios);

3) bus stops (mainly outdoor scenarios).

The sensing solutions to be adopted in these scenarios belong to the crowd monitoring/analysis [55] family. Detecting and estimating some features of a "crowd" in indoor and outdoor locations is an active research topic, with many applications, including surveillance and security, situation awareness, and emergencies (for a recent review, see [56] and references therein). In PT applications, the feature of interest is the number of components of the crowd (i.e., crowd counting) and/or its density (i.e., crowd density estimation); moreover, tracking of individuals in a crowd could be needed to build O-D matrices, useful for long-term planning. A review of crowd analysis techniques for urban applications, including transportation systems, is provided in [10], where approaches based on different data sources, including information fusion techniques, are discussed and compared.

Sensing technologies for crowd analysis can be classified [57] as visual-based (VB) solutions, based on still or moving images/videos mainly acquired by optical, thermal, or laser cameras, or non-visual based (NVB) ones, which do not rely on images to estimate crowd parameters, but resort to other physical quantities or features that can be related to crowd parameters, such as, e.g., those of radio signals, temperature, or sound.

To perform crowd analysis, VB techniques resort to sophisticated image processing, pattern recognition, or computer vision techniques $[55,58,59]$. Indeed, thanks to recent advances in AI, traditional camera sensors are becoming "smart" and can detect, recognize, and even identify persons. VB technologies perform passive sensing, relying on a network of dedicated sensors, without requiring active cooperation/participation of the users.

Among NVB technologies (see [60] for a recent review), sensing solutions based on mobile RF devices represent an interesting approach, due to the diffusion of smartphones and other portable/wearable devices, such as, e.g., pedometers, smart watches, or biometrical sensors. This approach to sensing is known as mobile sensing [61], opportunistic sensing [62] or participatory sensing [63]. Data collected by means of such device-aided NVB systems can be used not only to count people in a crowd, but also to gather additional information about individuals (e.g., planned routes, O-D flows, passengers using off-peak hours group ticket, and so on). However, a problem inherent to device-aided systems is that they usually require user cooperation/participation. To motivate participation, it is sometimes needed to introduce incentive or reward mechanisms, or apply radical modifications to the procedures to access the PT service, such as an authentication phase to use the service. Although this issue could raise privacy concerns, it could also be useful as a means to increase the overall safety of the PT systems during a pandemic outbreak, by reducing the risk that infected people can access the system.

When device-aided NVB techniques cannot be used for crowd characterization, due to, e.g., lack of user cooperation and/or security/privacy issues, RF-based non-device aided or device-free approaches can be pursued, which operate by analyzing the propagation channel variations of wireless signals induced by the people present in a given spatial area. In limited cases, NVB sensing techniques relying on physical properties different from those of RF signals (such as audio or ultrasound signals) can also be employed.

The main sensing technologies for crowd management in PT systems are summarized in Table I and will be discussed in the forthcoming subsections.

\section{A. Infrared sensors}

Infrared sensors (IR) are commonly used in traditional APC systems for counting the number of passengers boarding or alighting a vehicle (usually a bus or a tram). Commerciallyavailable solutions employ a couple of IR sensors (acting as a transmitter/receiver) forming a "light barrier" aimed at detecting the passage of people at the input/output gates of the vehicle. In alternative, a single pyrolectric IR (PIR) sensor can be used, which detects the IR radiation emitted by the human body in the wavelength range $2-14 \mu \mathrm{m}$. To enhance reliability, combined installation of IR/PIR sensors can also be conceived. Although IR sensors are very frequently used in PT systems, with counting accuracy generally well above $90 \%$ [64], their performances worsen when multiple people board through the same door simultaneously [65, 66]. Moreover, installation could be expensive, since typically more than one barrier per door is needed to detect the passenger flow.

\section{B. Pressure/load sensors}

Another solution commonly employed in traditional APC systems counts the number of passengers boarding or alighting buses or trams by means of pressure-sensitive switches ("treadle mats") placed on the steps, which are activated under the effect of the passenger weight. These solutions are simple, accurate (above $95 \%$ ) and rugged, ensuring long operational life: installation of multiple treadle mats over different steps allows one to discriminate between infeed/outfeed motion [4]. An innovative pressure sensor is the Velostat/Linqstat one, which is a carbon-impregnated conductive polymeric foil that can be used as a low-power inexpensive pressure sensor. Such a sensor has been adopted in [67] to implement a system aimed at monitoring seat occupancy in a bus.

Another application of pressure/load sensors, placed on the ground or on the suspensions, is in weigh-in-motion (WIM) systems, which estimate the number of passengers by the loading of the vehicle detected before and after the stops. Since most of the modern trains are equipped with electronic weighing sensors providing information to the braking system, a WIM solution exploiting such sensors has been proposed and implemented in the Copenaghen metro system [68]. In [69] two algorithms for passenger counting are proposed, which estimate the passenger load on the basis of the pressure variations of vehicle air ride suspensions, which are commonly 
TABLE I

A TAXONOMY OF SENSING TECHNOLOGIES FOR CROWD MANAGEMENT IN PT SYSTEMS.

\begin{tabular}{|c|c|c|c|c|c|c|c|c|c|}
\hline & Technology & Frequency & $\begin{array}{l}\text { Max } \\
\text { range }\end{array}$ & $\begin{array}{l}\text { Power } \\
\text { consumpt. }\end{array}$ & $\begin{array}{l}\text { User } \\
\text { cooper. }\end{array}$ & Accuracy & $\begin{array}{l}\text { Processing } \\
\text { complexity }\end{array}$ & Cost & Scenario \\
\hline \multirow{4}{*}{ 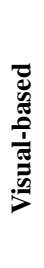 } & Optical & Visible & $100 \mathrm{~m}$ & Low & No & High & High & High & All \\
\hline & Thermal & Infrared & $500 \mathrm{~m}$ & Low & No & High & High & High & $\begin{array}{l}\text { Railway/metro station, } \\
\text { bus stop }\end{array}$ \\
\hline & LiDAR & $\begin{array}{l}\text { Ultraviolet, } \\
\text { Visible, } \\
\text { Near infrared }\end{array}$ & $1 \mathrm{~km}$ & Medium & No & High & $\begin{array}{l}\text { Medium- } \\
\text { high }\end{array}$ & Medium & $\begin{array}{l}\text { Railway/metro station, } \\
\text { bus stop }\end{array}$ \\
\hline & SAR & $\mathrm{Ku} / \mathrm{Ka}$ band & $10 \mathrm{~m}$ & Very high & No & High & High & High & Bus stop \\
\hline \multirow{12}{*}{ 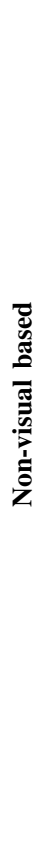 } & IR/PIR & Infrared & $10 \mathrm{~m}$ & Very low & No & High & Low & Medium & Bus/tram \\
\hline & Pressure & Mechanical & Contact & Very low & No & High & Low & Medium & Train and bus/tram \\
\hline & Acoustic & $0.01-100 \mathrm{kHz}$ & $20 \mathrm{~m}$ & Low & No & Low & Low & Low & $\begin{array}{l}\text { Train and bus/tram, } \\
\text { railway/metro station }\end{array}$ \\
\hline & IR-UWB & $3.1-10.6 \mathrm{GHz}$ & $100 \mathrm{~m}$ & Low & No & High & Low & Medium & $\begin{array}{l}\text { Railway/metro station, } \\
\text { bus stop }\end{array}$ \\
\hline & RSSI/CSI & Various & Variable & Very low & No & Medium & High & Low & $\begin{array}{l}\text { Railway/metro station, } \\
\text { bus stop }\end{array}$ \\
\hline & RFID & $13.56 \mathrm{MHz}$ & $10 \mathrm{~cm}$ & Very low & Yes & High & Low & Medium & $\begin{array}{l}\text { Train and bus/tram, } \\
\text { Railway/metro station }\end{array}$ \\
\hline & $\mathrm{NFC}$ & $13.56 \mathrm{MHz}$ & $10 \mathrm{~cm}$ & Very low & Yes & High & Low & Medium & $\begin{array}{l}\text { Train and bus/tram, } \\
\text { Railway/metro station }\end{array}$ \\
\hline & Bluetooth & $2.4 \mathrm{GHz}$ & $50 \mathrm{~m}$ & Very low & Yes & Medium & Low & Low & $\begin{array}{l}\text { Railway/metro station, } \\
\text { bus stop }\end{array}$ \\
\hline & WiFi & $2.4 / 5 \mathrm{GHz}$ & $100 \mathrm{~m}$ & Medium & Yes & Low & Medium & Low & $\begin{array}{l}\text { Railway/metro station, } \\
\text { bus stop }\end{array}$ \\
\hline & LTE & $\begin{array}{l}800 / 1800 / 2600 \\
\mathrm{MHz}\end{array}$ & $10 \mathrm{~km}$ & High & Yes & Low & High & Medium & $\begin{array}{l}\text { Railway/metro station, } \\
\text { bus stop }\end{array}$ \\
\hline & $5 \mathrm{G}$ & Sub-6 GHz & $10 \mathrm{~km}$ & High & Yes & Low & Low & Medium & $\begin{array}{l}\text { Railway/metro station, } \\
\text { bus stop }\end{array}$ \\
\hline & $5 \mathrm{G}$ & MMW band & $1 \mathrm{~km}$ & High & Yes & Medium & Low & Medium & $\begin{array}{l}\text { Railway/metro station, } \\
\text { bus stop }\end{array}$ \\
\hline
\end{tabular}

${ }^{\text {a }}$ Power consumption refer to a single sensor.

employed in almost all modern transit buses. WIM systems represent a convenient solution to measure crowdedness inside vehicles, even though it can be difficult to infer the actual number of passengers boarding or alighting [4].

\section{Optical cameras}

Optical cameras are widely used in private and public spaces for surveillance and security, like in closed circuit television (CCTV) systems, and are routinely installed inside PT vehicles and stations to this aim. Since they can often support crowd monitoring functions with firmware/software upgrades, optical cameras are among the most versatile and used techniques for crowd analysis [58, 70]. Most works on crowd counting and detection based on optical cameras rely on computer-vision technologies $[65,71,72]$, wherein crowds are detected using specific features, e.g., facial recognition or motion tracking, extracted from images/videos. Moreover, the majority of recent works employ AI or ML/DL algorithms [73].

Camera-based solutions can be applied in all transportation scenarios, both indoor and outdoor ones. Video cameras mounted inside road or rail vehicles can be used to estimate the number of passengers and their flow (i.e., whether they are boarding or alighting) [74, 65, 75, 76]. An image-processing technique based on a modified Hough transform is proposed in [74], aimed at detecting the contour features of heads and estimating accordingly the number of passengers and their flow in a bus. Many recent approaches are based on convolutional neural networks (CNNs), such as the passenger counting system proposed in [77], which exploits also the spatio-temporal properties of video sequences acquired on a PT bus in China, or the solution proposed in [78], where crowd density inside a bus is detected and classified in 5 different levels (from very low to very high), to be displayed by LCD screens installed at the bus stops. A deeply-recursive CNNbased solution is proposed in [79] and tested on a dataset of images taken at the Bus Rapid Transit (BRT) in Bejing, China. A neural-network based crowd density estimation algorithm is described in [80], targeted at underground station platforms, which has been experimentally tested on a sequence of CCTV images acquired at a metro station in Hong Kong. Finally, VB techniques for counting people at bus stops are proposed in $[81,82]$, based on computer-vision techniques, which process measurements of foreground areas corrected by suitable perspective transformations.

In summary, crowd analysis based on optical sensors is versatile and powerful, but has several limitations: camera 
sensors, indeed, are expensive, and each camera can only cover a small area, resulting in high deployment costs in complex and/or large environments. In some scenarios, optical camerabased systems do not allow to estimate the number of people with sufficient accuracy, due to possible obstructions, clutter, and poor light/weather conditions. Moreover, crowd analysis techniques based on images require a high computing power. Finally, cameras capture a great deal of personal information, with related privacy concerns.

\section{Thermal cameras}

Thermal cameras can detect people in low-light environments, complete darkness, or other challenging conditions, such as smoke-filled and dusty environments [83]. Thermal imaging cameras are currently used in some countries to prevent accidents and infrastructure damage in PT systems, detecting, for example, people walking on the tracks or fire. This technology, however, can be employed also to monitor crowding situations both in indoor and outdoor scenarios.

Some people counters based on thermal cameras are proposed in $[84,85,86]$. Different from optical cameras, people counters based on thermal cameras are less sensitive to the level of ambient lighting or background colour contrasts, but their performance can be adversely affected by heat sources and weather conditions. In addition, real-time image processing can be computationally intensive. The high costs of instrumentation still limit the widespread use of thermography for crowd monitoring.

Recently, hybrid approaches, combining thermal and optical imaging sensors, and intelligent processing (based on DL and $\mathrm{CNN}$ ), have been implemented to improve the accuracy and real-time processing of camera-based systems [87], [88]. In [89], a people counting algorithm is developed, which uses low-resolution thermal images and employs small-size CNNs, being able to run on a limited-memory and low-power platform.

\section{E. LiDAR}

Another option to detect and track persons is represented by light detection and ranging (LiDAR) sensors, especially in environments where there are several interacting people [90]. A LiDAR sensor is a distance measuring system that works by illuminating the target with a laser beam and sensing the reflected laser light. There are different implementations of LiDAR sensors based on their coverage area and wavelengths.

Compared to traditional VB approaches, LiDAR is less sensitive to varying lighting conditions and requires, in general, lower data-processing time. Thanks to these features, LiDARbased counting systems are suitable to all transportation scenarios, both indoor and outdoor.

A real-time 2D LiDAR monitoring system for people counting is proposed in [91], which turns out to be useful in monitoring wide areas and dense groups of persons. The solution proposed in [92], instead, employs two LiDAR sensors set at different heights, aimed at detecting people's heads and knees, to improve tracking performance. To increase system accuracy, other solutions use three-dimensional (3D) LiDAR: in [93], for example, a technique for people counting is presented, which works well even if two or three persons pass at the same time. However, an important limitation of 3D LiDAR is represented by its computing cost, which can be even higher than optical camera-based solutions.

\section{F. Acoustic/ultrasound sensors}

Acoustic sensor-based approaches perform people counting by relying on audio signals transmitted by smartphones or produced by speaking people [60]. A crowd counting solution based on audio tones is presented in [94], leveraging the microphones and speaker phones available in most mobile phones. Effectiveness is proven through several experiments at bus stops or aboard, which show, however, that counting latency can significantly grow as the number of devices increases; as a consequence, this technique may be appropriate only for low-density scenarios.

Despite their simplicity, applicability of purely acousticbased solutions in crowd counting is largely limited by their high sensitivity to environmental noise level. For this reason, hybrid solutions, employing not only acoustic sensors, have been proposed. In [95], for example, a multimodal sensor network is built, which exploits sound intensity in conjunction with additional information sources, like carbon-dioxide level and RF link strength, in order to increase estimation accuracy. Another hybrid solution is presented in [96], which proposes an opportunistic collaborative sensing system, based on acoustic and motion sensors integrated in smartphones.

A different option to count people is represented by acoustic-based solutions using ultrasound sensors, which perform well for indoor spaces and small crowds [60]. When the reverberation of the transmitted waves is received, the number of people can be estimated by exploiting information from the receive time or the signal decay. Based on this approach, an ultrasonic sensing technique for estimating the number of people is presented in [97], which exhibits satisfactory performance when the occupancy of the space does not reach its maximum.

\section{G. Device-free RF sensing}

Device-free crowd analysis based on RF signals is an emerging technique, which does not require installation of expensive cameras nor suffer from privacy related concerns. It exploits the impact of the monitored crowd on RF communications to infer information regarding the size and/or density of the crowd, either using traditional radar methodologies (range and Doppler analysis) $[98,99,100]$, mainly with impulse-radio (IR) ultrawideband (UWB) signals $[66,101]$ or by analyzing features extracted by channel quality measurements, such as received signal strength indicator (RSSI) [102, 103, 104] or channel state information (CSI) [105, 106, 107].

People counting using RF signals can be used in dimly lit places, and in smoky and dusty environments: hence, it represents an interesting solution for subway stations and/or at bus stops. In [66] a people counting algorithm using a couple of IR-UWB radar sensors is proposed, which was tested in a subway platform in Seoul, South Korea, showing accuracy 
values higher than $90 \%$. A solution to count the people in a queue, based on RSSI measurements carried out by Bluetooth low-energy (BLE) ${ }^{1}$ devices, is described in [108]: the system is completely passive and estimates the number of persons in the queue by analyzing the mean and variance of the RSSI values between a BLE beacon and a receiver covering a certain area.

In general, RSSI-based approaches for crowd analysis exhibit good performance in small monitored environments, where the propagation channel variations are dominated by the attenuation caused by the people actually present in the environment. On the other hand, in a rich scattering environment, crowd analysis approaches based on CSI provide a more reliable people counting (but are considerably more complex). Belonging to this class, in [109] and [106] the received WiFi and Long Term Evolution (LTE) downlink control signals are processed, respectively, to extract the changes of the propagation channel induced by the presence of different number of people: the related counting algorithms exhibit variable levels of accuracy in different scenarios.

Finally, more sophisticated microwave SAR tomography techniques $[47,110]$ can also be used, which provide specific $\mathrm{RF}$ images from which more detailed crowd information can be extracted, by using complex image classification algorithms (i.e., count, distribution, mobility, etc.). Modeling issues induced by the intrinsic near-field scenario (e.g., typical for bus stops) could be overcome using specialized algorithms [111].

In summary, excluding SAR-based techniques, device-free RF sensing is a moderate-complexity crowd monitoring technique with low installation costs. Moreover, RF signals can penetrate obstacles to a certain extent and are not affected by weather/illumination. A moderate accuracy (around $80 \%$ for RSSI-based techniques, up to $90 \%$ and larger for CSIbased ones) can be expected in simple scenarios, but it is questionable whether this approach can be scalable to large crowds, especially in complex propagation scenarios [60]. Moreover, counting algorithms based on this approach usually require a site-specific training phase, which complicates practical installation and maintenance.

\section{H. Device-aided RF sensing}

In device-aided approaches for crowd analysis, users are expected to carry RF devices, which must be switched on to enable people counting. Since modern smartphones are ubiquitous and, moreover, are equipped with several sensors and multiple RF interfaces, device-aided solutions are commonly used to gather different types of information for many different purposes and applications (see [60] for a recent review).

1) RFID and NFC: RFID-based solutions require that the passengers carry passive RFID tags, whose presence can be detected by a reader. The Authors in [112] propose an APC system for bus vehicles employing commercial EPC Gen2 tags, which are recognized by a reader located in correspondence of the bus gate. A similar short-range communication system is Near Field Communication (NFC) [113] technology,

\footnotetext{
${ }^{1} \mathrm{BLE}$ is a low-energy consumption version of Bluetooth standard, which assures better communication performances with a limited power consumption
}

which is currently supported by many modern smartphones and tablets. When a device with NFC functionalities appears in the reader's working range, which can be placed at the station gates or at any other fixed access point, it "wakes up" and sends a signal containing encoded data. Finally, it should be observed that RFID and NFC technologies are used in traditional and emerging AFC systems for electronic ticketing, such as MIFARE contactless cards or mobile-based payment systems [114], which can also be employed for passenger counting.

2) Bluetooth: Bluetooth is a consolidated short-range RF technology, supported by almost all smartphones on the market: crowd monitoring algorithms using Bluetooth have been proposed in several papers (see e.g. [115] and reference therein). An algorithm based on off-the-shelf Bluetooth hardware for counting bus passengers has been proposed in [116] and tested in the city of Funchal, Portugal. The system consists of a Bluetooth scanner mounted on the ceiling of a bus, which periodically scans for discoverable Bluetooth devices in its range, and is aimed at discovering O-D relations by postprocessing data and correlating them with information related to bus location and tickets issued by fare machines.

Bluetooth can also represent an efficient solution for crowd counting at bus stops. A crowd analysis solution based on BLE is proposed in [117], where a large population carry BLE proximity tags, acting as beacons, whose presence is sensed by smartphone carried by few volunteers. A reciprocal solution can be used at bus stops, where BLE beacons are installed at the bus stations, and are detected by passenger smartphones in close proximity of the stops. ${ }^{2}$

Compared to WiFi-based sensing (discussed later), Bluetooth devices are cheaper, less power-hungry and are characterized by increased flexibility and simplified installation.

3) WiFi: Many crowd analysis solutions exploit the characteristics of the IEEE 802.11 (WiFi) protocol, which is widely used by passengers during their trips. The technique adopted in $[18,20]$ estimates the current number of passengers in a bus by counting the number of probe requests, i.e., medium access control (MAC) addresses, sent by WiFi-equipped devices in the vehicle. A similar approach is followed in [119], where a de-randomization mechanism is introduced to counteract software randomization of MAC addresses, recently introduced in many operating systems.

One of the problem inherent to the use of WiFi-based techniques for crowd counting inside vehicles is the ability to distinguish between people outside the vehicle and actual passengers. This issue was tackled in $[18,20]$ by filtering the probes with a sliding window, aimed at removing MAC addresses that were not detected over a longer period of time. In addition, in [119] the received power is also used to discriminate devices that are likely to be outside the bus. The system in $[18,20]$ was able to detect only around $20 \%$ of the passengers in a real setting, since several users may have turned off the WiFi interface. Underestimation of the number

\footnotetext{
${ }^{2}$ This solution relies on the same technology introduced by Google and Apple in the most recent versions of their smartphone operating systems, and is used by many national contact-tracing apps (e.g., Immuni for Italy [118]).
} 
of passengers is a common problem for these techniques, which can be compensated by a proper calibration of the procedure in each scenario of interest.

The main advantage of WiFi-based techniques is that they allow to track passengers also when they alight the bus, allowing to estimate O-D flows. WiFi-based counting can also be employed in metro/railway stations, since access points are typically available in such scenarios.

4) Cellular: Similarly to WiFi, cellular signals such as LTE and $5 \mathrm{G}$ can be used for device-aided crowd counting, thanks to their ubiquitous availability and good penetration in indoor environments. Cellular signals could be available in areas where the WiFi coverage is not present, such as bus stops, remote and small railways/metro stations. A cellular-based crowd density estimation method is proposed in [120], which measures the signal strength emitted in uplink by the smartphones of the crowd components, and classifies the crowd density in different levels using deep learning techniques, with an accuracy of $78 \%$ when three levels are considered.

In principle, the position of users in a cellular network can be obtained with satisfactory precision by combining knowledge of the serving base station, RSSI values, and triangulation principles [121], which can be at the basis for large-scale crowd analysis. However, this approach is difficult to be used in real-time, whereas it is more suited for long-term travel demand estimation [122]. Real-time passenger counting using cellular data is rarely performed, due to several drwbacks: (i) it requires gathering data from different mobile operators; (ii) it raises significant privacy concerns; (iii) it does not allow one to precisely discriminate passengers from general public in open areas. A breakthrough could be the planned introduction in $5 \mathrm{G}$ systems of the millimeter-wave (MMW) band, which will require very small cells: from the viewpoint of crowd analysis, the placement of small cells allows one to more precisely monitor spatially limited areas, like bus stops.

\section{Discussion}

In this Section, we provide a final discussion regarding the applications of the above-mentioned sensing techniques in the different transportation scenarios. ${ }^{3}$

1) Trains/buses/trams: Such vehicular scenarios are characterized by a well delimited indoor space with a limited number of accesses (gates). Traditional APC systems [4, 35, 119] count the number of passengers inside vehicles on the basis of various onboard sensors, mainly IR or pressure-sensitive ones. The number of passengers can also by estimated by the number of validated/issued tickets, as in AFC systems, which however requires user cooperation and can provide underestimated results in case of diffuse fare evasion.

Although solutions based on sensors installed on the vehicles are simple, they require a large initial investment. Therefore, the diffusion of portable and mobile devices between passengers have pushed many researchers to study solutions based on RF techniques (both device-aided and device-free).

\footnotetext{
${ }^{3}$ For a review and discussion of some industrial solutions employing some of these technologies for people counting in PT systems, see [60].
}

Summarizing, as also indicated in Table I, even though more sophisticated VB/NVB sensing technologies may be used as well, their usage is not expected to lead to significant innovations in this scenario, compared to traditional solutions adopted in commercial APC systems.

2) Railway/metro stations: Many crowd monitoring options are available in this scenario, since the access to the stations occurs through a limited number of gates. Morover, CCTV surveillance systems are typically present inside stations and can be used for VB crowd analysis. The access to train platforms is usually governed by turnstiles where tickets/passes must necessarily be validated: thus, IR-based APC or AFC systems could be a reasonable option to count passengers in this scenario. However, this solution only counts people trying to access the platforms, disregarding other people which could walk inside the station for different purposes (e.g., for shopping or leisure). RF-based techniques could suffer from coverage problems, especially within metro/underground stations.

3) Bus stops: This scenario is by far the most difficult to manage, since bus stops are usually located in outdoor spaces, not well delimited by fixed gates. In this case, both VB and NVB sensing technologies can be used, but it is imperative to adopt cost-effective, rugged, and low-power solutions, in order to reduce the maintenance cost. Moreover, solutions that do not require significant infrastructures are preferred, since in many cases the bus stops are not equipped with shelters and are indicated by simple poles.

\section{MAIN INNOVATIONS AND ADVANTAGES}

The crowd management functionalities of the new framework can provide several innovations and advantages that are not present in legacy ITSs:

1) Proactive control of station access: In railway/subway applications, on the basis of the knowledge of the number of passengers aboard the arriving trains and the prediction of those alighting at the station, it will be possible to predict the number of accesses to stations/platforms with a low error margin and in real-time, so as to avoid crowding. This number can be communicated to the users (by displays at the station gates or by the mobile transport apps) and can be used by the security operators to filter passengers at the turnstiles. Priority policies can be envisioned, such as taking into account the time already spent in queue, or the trip motivations (e.g., a priority could be assured to health workers, disabled or elder users, law enforcement, and teachers/students).

2) Vehicle access reservation: In bus/tram trips, a vehicle access reservation system can be implemented. A sensor at the bus stop detects the user presence and exchanges information with his/her device (i.e., the smartphone), so as to grant him/her the access to board the first arriving bus (a virtual queuing system) or putting him/her in an overbooking list (with priority) to allow him/her to board the next one. The application can generate an e-ticket with the access grant (e.g., a QR-code) that can be validated on board at the ticket machine. 
TABLE II

MAIN ADVANTAGES RELATED TO ADOPTION OF CROWD MANAGEMENT IN PT SYSTEMS.

\begin{tabular}{ll}
\hline Actor & Advantages \\
\hline PT service operator (manager) & $\begin{array}{l}\text { Having real-time crowding data of the different segments of the PT system } \\
\text { allows one to plan services more efficiently and to quickly readapt them to } \\
\text { tackle critical situations, localized in space and time }\end{array}$ \\
PT operators (drivers, inspectors, etc.) & $\begin{array}{l}\text { The possibility to know in advance crowding situations at the bus stops or } \\
\text { stations allows one to tackle critical situations (planning for examples possible } \\
\text { holding, stop-skipping, or alternative routes) }\end{array}$ \\
Users & $\begin{array}{l}\text { Knowing crowding situations allows one to use alternative means or to make } \\
\text { the trip in another hours, if not strictly necessary; by the reservation system, } \\
\text { if available, the users can access vehicles without unnecessary crowding at the } \\
\text { bus stops or stations }\end{array}$ \\
Police forces & $\begin{array}{l}\text { Knowing in real-time and/or predicting possible crowds - potentially dangerous } \\
\text { for public health and/or for public order - allows one a more timely and targeted } \\
\text { intervention }\end{array}$ \\
Sanitary system & $\begin{array}{l}\text { Smart reduction of crowding resulting from an agile management of the PT } \\
\text { system allows one to reduce the diffusion of infection and prevent further } \\
\text { outbreaks }\end{array}$ \\
Reduction of private car usage, less pollution
\end{tabular}

3) Crowding information dissemination: Users receive realtime information related to available capacity (in terms of number of seats or in percentage) of the bus/trains in arrival and/or crowding at bus stops/stations by means of displays installed in correspondence of the bus stops or at the entrance of the stations, or by messages/alerts issued by mobile transport apps, so as to avoid unnecessary waiting or crowding, and possibly reschedule their trips.

4) Crowd-aware route planning: Users can plan their trips on the mobile transport app, by taking into account not only geographical information and traveling times (static data), but also traffic and crowding information about vehicles and bus stops/stations during the trip (dynamic data). The app may not necessarily suggest the shortest route, but the least crowded one, taking into account also crowding levels measured during the trip. Such a feature not only help reduce crowds (and the consequent infection risk in case of a pandemic), but also distribute more efficiently the load on the transportation network.

The main advantages of the crowd management framework are summarized in Table II. Compared to static methods, like traditional survey-based compilation of O-D matrix flows, more efficient planning and real-time control of the operation of the PT system is allowed. The large amount of generated data can be used by AI and ML/DL algorithms to better understand and plan a series of aspects generally associated with improvements of the quality of life in urban areas and smart cities.

Compared to other anti-COVID-19 solutions (see Section II), the new framework is not aimed at enforcing social distancing measures only. Its scope is wider, since it tries to optimize the overall performance of the PT system. As a byproduct, it also allows to partially recover the drawbacks and inefficiencies of PT systems due to the adoption of rigid social distancing measures during both pandemic and post-pandemic phases.

\section{CONCLUSIONS}

A clean, smart, and resilient PT system is at the core of worldwide economies and is central to people's lives: this is why there has been an increased number of research articles that discuss the feasibility of integrating ICT/IoT sensing solutions in ITSs. In this paper, we have reviewed the main ICT/IoT sensing technologies for crowd analysis, showing also how they can be adopted in a reference architecture aimed at introducing innovative crowd management functionalities in legacy ITS systems. The new framework is based on some basic components and subsystems, which can be used as building blocks to implement an evolved ITS, capable of real-time monitoring and predicting crowd situations, as well as disseminating useful information to users at the bus stops/stations and/or through mobile transport apps.

Some features of the new framework are similar to those of a contact tracing system, which can be implemented more easily by resorting to user cooperation. In this sense, the crowd detection functionalities can be incorporated in a more complex system, which can implement, besides the typical mobile transport app functionalities (like Moovit), also the possibility to buy tickets and/or to reserve the access to the vehicles, in conditions of particular crowding. This could represent a decisive incentive to the use of a PT system. However, the more appropriate crowd monitoring solution must be singled out case-by-case, in dependence on the scenario, the ICT infrastructure owned or leased by the PT operator, the socioeconomic context, and the cost-benefits ratio. The potentials of crowd management go beyond the scope of dealing with typical social distancing problems, by potentially allowing real-time optimization and management of the PT system.

\section{ACKNOWLEDGMENT}

The authors would like to thank the IEEE ComSoc/VTS Italian Chapter for supporting this work, by awarding the first prize to the SALUTARY (Safe and Reliable Public 
Transportation System) system concept within the call for ideas in response to COVID-19 outbreak in Italy.

\section{REFERENCES}

[1] European Commission, EUROPEAN MOBILITY WEEK 2021: Safe and healthy with sustainable mobility, 2021. [Online]. Available: https : / / mobilityweek.eu.

[2] G. Dimitrakopoulos, L. Uden, and I. Varlamis, The Future of Intelligent Transport Systems, G. Dimitrakopoulos, L. Uden, and I. Varlamis, Eds. Elsevier, 2020. [Online]. Available: https://www.sciencedirect. com/science/article/pii/B9780128182819000279.

[3] N. B. Hounsell, B. P. Shrestha, and A. Wong, "Data management and applications in a world-leading bus fleet," Transportation Research Part C: Emerging Technologies, vol. 22, pp. 76-87, 2012. [Online]. Available: https://www. sciencedirect.com/science/ article/pii/S0968090X11001707.

[4] I. Pinna, B. Dalla Chiara, and F. P. Deflorio, "Automatic passenger counting and vehicle load monitoring," Ingegneria Ferroviaria, vol. 65, no. 2, pp. 101$138,2010$.

[5] C. N. Opurum, Automated Fare Collection System \& Urban Public Transportation: An Economic \& Management Approach to Urban Transit Systems. Trafford Publishing, 2012. [Online]. Available: https://books. google.it/books?id=6uoXmf1J2ioC.

[6] C. Iliopoulou and K. Kepaptsoglou, "Combining ITS and optimization in public transportation planning: State of the art and future research paths," European Transport Research Review, vol. 11, no. 1, p. 27, May 2019. [Online]. Available: https://doi.org/10.1186/ s12544-019-0365-5.

[7] H. Habibzadeh, Z. Qin, T. Soyata, and B. Kantarci, "Large-scale distributed dedicated- and non-dedicated smart city sensing systems," IEEE Sensors Journal, vol. 17, no. 23, pp. 7649-7658, 2017.

[8] G. Cardone, A. Cirri, A. Corradi, L. Foschini, R. Ianniello, and R. Montanari, "Crowdsensing in urban areas for city-scale mass gathering management: Geofencing and activity recognition," IEEE Sensors Journal, vol. 14, no. 12, pp. 4185-4195, 2014.

[9] D. S. Gallo, C. Cardonha, P. Avegliano, and T. C. Carvalho, "Taxonomy of citizen sensing for intelligent urban infrastructures," IEEE Sensors Journal, vol. 14, no. 12, pp. 4154-4164, 2014.

[10] M. S. Kaiser et al., "Advances in crowd analysis for urban applications through urban event detection," IEEE Transactions on Intelligent Transportation Systems, vol. 19, no. 10, pp. 3092-3112, 2018.

[11] X. Sheng, J. Tang, X. Xiao, and G. Xue, "Sensing as a service: Challenges, solutions and future directions," IEEE Sensors Journal, vol. 13, no. 10, pp. 3733-3741, 2013.
[12] M. R. Palattella et al., "Internet of Things in the 5G Era: Enablers, Architecture, and Business Models," IEEE Journal on Selected Areas in Communications, vol. 34, no. 3, pp. 510-527, 2016.

[13] F. Guo, F. R. Yu, H. Zhang, X. Li, H. Ji, and V. C. M. Leung, "Enabling Massive IoT Toward 6G: A Comprehensive Survey," IEEE Internet of Things Journal, vol. 8, no. 15, pp. 11891-11915, 2021.

[14] M. Cantwell, B. Caulfield, and M. O'Mahony, "Examining the factors that impact upon public transport commuting stress," Journal of Public Transportation, vol. 12, no. 2, pp. 1-21, 2009.

[15] A. Tirachini, D. A. Hensher, and J. M. Rose, "Crowding in public transport systems: Effects on users, operation and implications for the estimation of demand," Transportation Research Part A: Policy and Practice, vol. 53, pp. 36-52, 2013. [Online]. Available: https : / www. sciencedirect. com/science/article/pii / S0965856413001146.

[16] A. Drabicki, O. Cats, and R. Kucharski, "The potential of real-time crowding information in reducing bus bunching under different network saturation levels," in 2021 7th International Conference on Models and Technologies for Intelligent Transportation Systems (MT-ITS), 2021, pp. 1-6.

[17] G. E. Sánchez-Martínez, H. N. Koutsopoulos, and N. H. M. Wilson, "Real-time holding control for high-frequency transit with dynamics," Transportation Research Part B: Methodological, vol. 83, pp. 1-19, 2016. [Online]. Available: http://www. sciencedirect. com/science/article/pii/S019126151500257X.

[18] M. Handte et al., "Crowd density estimation for public transport vehicles," in Proceedings of the Workshops of the EDBT/ICDT 2014 Joint Conference (EDBT/ICDT 2014), Athens, Greece, March 28, 2014, vol. 1133, 2014, pp. 315-322. [Online]. Available: http://ceurws.org/Vol-1133/paper-51.pdf.

[19] K. Farkas, G. Feher, A. Benczur, and C. Sidlo, "Crowdsending based public transport information service in smart cities," IEEE Communications Magazine, vol. 53, pp. 158-165, Aug. 2015.

[20] M. Handte, S. Foell, S. Wagner, G. Kortuem, and P. J. Marrón, "An Internet-of-Things enabled connected navigation system for urban bus riders," IEEE Internet of Things Journal, vol. 3, no. 5, pp. 735-744, Oct. 2016.

[21] P. Wang, X. Chen, Y. Zheng, L. Cheng, Y. Wang, and D. Lei, "Providing real-time bus crowding information for passengers: A novel policy to promote high-frequency transit performance," Transportation Research Part A: Policy and Practice, vol. 148, pp. 316-329, 2021. [Online]. Available: https : // www . sciencedirect . com / science / article / pii / S0965856421001051.

[22] A. Boukerche and R. W. L. Coutinho, "Crowd management: The overlooked component of smart transportation systems," IEEE Communications Magazine, vol. 57, no. 4, pp. 48-53, 2019. 
[23] T. Franke, P. Lukowicz, and U. Blanke, "Smart crowds in smart cities: Real life, city scale deployments of a smartphone based participatory crowd management platform," Journal of Internet Services and Applications, vol. 6, no. 1, p. 27, 2015. [Online]. Available: https://doi.org/10.1186/s13174-015-0040-6.

[24] A. Jolfaei, V. G. Menon, C. Lv, A. K. Bashir, Y. K. Tan, and K. Kant, "Guest editorial advanced sensing and sensor fusion for intelligent transportation systems," IEEE Sensors Journal, vol. 21, no. 14, pp. 15 425-15 426, 2021.

[25] H. Lian, X. Pei, and X. Guo, "A local environment model based on multi-sensor perception for intelligent vehicles," IEEE Sensors Journal, vol. 21, no. 14, pp. 15 427-15 436, 2021.

[26] L. Yang et al., "A refined non-driving activity classification using a two-stream convolutional neural network," IEEE Sensors Journal, vol. 21, no. 14, pp. 15 574-15 583, 2021.

[27] V. Singh and N. K. Verma, "Intelligent condition-based monitoring techniques for bearing fault diagnosis," IEEE Sensors Journal, vol. 21, no. 14, pp. 15448 $15457,2021$.

[28] Y. Yan, H. Ma, M. Wen, S. Dang, and H. Xu, "Multifeature fusion-based mechanical fault diagnosis for onload tap changers in smart grid with electric vehicles," IEEE Sensors Journal, vol. 21, no. 14, pp. $15696-$ 15 708, 2021.

[29] W. Ejaz et al., "IoV-Based Deployment and Scheduling of Charging Infrastructure in Intelligent Transportation Systems," IEEE Sensors Journal, vol. 21, no. 14, pp. 15 504-15 514, 2021.

[30] F. Kortmann et al., "Modeling the quarter-vehicle: Use of passive sensor data for road condition monitoring," IEEE Sensors Journal, vol. 21, no. 14, pp. 15535 $15543,2021$.

[31] R. Mishra, H. P. Gupta, and T. Dutta, "A road health monitoring system using sensors in optimal deep neural network," IEEE Sensors Journal, vol. 21, no. 14, pp. 15 527-15 534, 2021.

[32] C. Chen, Y. Zhang, M. R. Khosravi, Q. Pei, and S. Wan, "An intelligent platooning algorithm for sustainable transportation systems in smart cities," IEEE Sensors Journal, vol. 21, no. 14, pp. 15437-15447, 2021.

[33] H. Peng, Y. Wang, Z. Chen, and Z. Lv, "Dynamic sensor speed measurement algorithm and influencing factors of traffic safety with wireless sensor network nodes and RFID," IEEE Sensors Journal, vol. 21, no. 14, pp. 15679-15686, 2021.

[34] R. Wang, F. Xie, J. Zhao, B. Zhang, R. Sun, and J. Yang, "Smartphone sensors-based abnormal driving behaviors detection: Serial-feature network," IEEE Sensors Journal, vol. 21, no. 14, pp. 15719-15728, 2021.

[35] I. Grgurević, K. Juršić, and V. Rajič, "Review of automatic passenger counting systems in public urban transport," in 5th EAI International Confer- ence on Management of Manufacturing Systems, L. Knapčíková, D. Peraković, A. Behúnová, and M. Periša, Eds., Cham: Springer International Publishing, 2022, pp. 1-15.

[36] Ipsos, Impact of coronavirus to new car purchase in China, Mar. 2020. [Online]. Available: https://www. ipsos.com/en/impact-coronavirus-new-car- purchasechina.

[37] A. Gutiérrez, D. Miravet, and A. Domènech, "COVID19 and urban public transport services: Emerging challenges and research agenda," Cities \& Health, pp. 1-4, 2020. [Online]. Available: https://doi.org/10.1080/ 23748834.2020 .1804291$.

[38] A. Aloi et al., "Effects of the COVID-19 lockdown on urban mobility: Empirical evidence from the city of Santander (Spain)," Sustainability, vol. 12, no. 9, p. 3870, 2020. [Online]. Available: https://doi.org/10. 3390/su12093870.

[39] T. Campisi, S. Basbas, A. Skoufas, N. Akgün, D. Ticali, and G. Tesoriere, "The Impact of COVID-19 Pandemic on the Resilience of Sustainable Mobility in Sicily," Sustainability, vol. 12, no. 21, 2020. [Online]. Available: https://www.mdpi.com/2071-1050/12/21/ 8829.

[40] A. Tirachini and O. Cats, "COVID-19 and public transportation: Current assessment, prospects, and research needs," Journal of Public Transportation, vol. 1, no. 22, pp. 1-21, 2020.

[41] P. Coppola and F. De Fabiis, "Evolution of mobility sector during and beyond Covid-19 emergency: A viewpoint of industry consultancies and public transport companies," TeMA - Journal of Land Use, Mobility and Environment, pp. 81-90, Jun. 2020. [Online]. Available: http://www.serena.unina.it/index.php/tema/ article/view/6900.

[42] V. Chamola, V. Hassija, V. Gupta, and M. Guizani, "A comprehensive review of the COVID-19 pandemic and the role of IoT, drones, AI, blockchain, and $5 \mathrm{G}$ in managing its impact," IEEE Access, vol. 8, pp. 90225 90265, 2020.

[43] C. T. Nguyen et al., "A comprehensive survey of enabling and emerging technologies for social distancing -Part I: Fundamentals and enabling technologies," IEEE Access, vol. 8, pp. 153 479-153 507, 2020.

[44] C. T. Nguyen et al., "A comprehensive survey of enabling and emerging technologies for social distancing - Part II: Emerging technologies and open issues," IEEE Access, vol. 8, pp. 154 209-154 236, 2020.

[45] K. Gkiotsalitis and O. Cats, "Public transport planning adaption under the COVID-19 pandemic crisis: Literature review of research needs and directions," Transport Reviews, vol. 41, no. 3, pp. 374-392, 2021. [Online]. Available: https://doi.org/10.1080/01441647. 2020.1857886.

[46] S. M. Asad, K. Dashtipour, S. Hussain, Q. H. Abbasi, and M. A. Imran, "Travelers-tracing and mobility profiling using machine learning in railway systems," in 
2020 International Conference on UK-China Emerging Technologies (UCET), 2020, pp. 1-4.

[47] A. Reigber, E. Alivizatos, A. Potsis, and A. Moreira, "Extended wavenumber-domain synthetic aperture radar focusing with integrated motion compensation," IEE Proceedings - Radar, Sonar and Navigation, vol. 153, no. 3, pp. 301-310, 2006.

[48] M. M. Rahman, K. C. Paul, M. A. Hossain, G. G. M. N. Ali, M. S. Rahman, and J.-C. Thill, "Machine learning on the COVID-19 pandemic, human mobility and air quality: A review," IEEE Access, vol. 9, pp. 72420-72 450, 2021.

[49] A. Drabicki, R. Kucharski, O. Cats, and A. Fonzone, "Simulating the effects of real-time crowding information in public transport networks," in 2017 5th IEEE International Conference on Models and Technologies for Intelligent Transportation Systems (MT-ITS), 2017, pp. 675-680.

[50] A. Nuzzolo, U. Crisalli, A. Comi, and L. Rosati, "A mesoscopic transit assignment model including real-time predictive information on crowding," Journal of Intelligent Transportation Systems, vol. 20, no. 4, pp. 316-333, 2016.

[51] J. Zhang et al., "A real-time passenger flow estimation and prediction method for urban bus transit systems," IEEE Transactions on Intelligent Transportation Systems, vol. 18, no. 11, pp. 3168-3178, 2017.

[52] T. Arabghalizi and A. Labrinidis, "Data-driven bus crowding prediction models using context-specific features," ACM/IMS Trans. Data Sci., vol. 1, no. 3, Sep. 2020. [Online]. Available: https://doi.org/10.1145/ 3406962.

[53] E. Jenelius, "Data-driven metro train crowding prediction based on real-time load data," IEEE Transactions on Intelligent Transportation Systems, vol. 21, no. 6, pp. 2254-2265, 2020.

[54] E. Jenelius, "Personalized predictive public transport crowding information with automated data sources," Transportation Research Part C: Emerging Technologies, vol. 117, p. 102647, 2020. [Online]. Available: https ://www. sciencedirect.com/science/article/pii/ S0968090X20305623.

[55] B. Zhan, D. N. Monekosso, P. Remagnino, S. A. Velastin, and L.-Q. Xu, "Crowd analysis: A survey," Machine Vision and Applications, vol. 19, no. 5-6, pp. 345-357, 2008.

[56] M. Bendali-Braham, J. Weber, G. Forestier, L. Idoumghar, and P.-A. Muller, "Recent trends in crowd analysis: A review," Machine Learning with Applications, vol. 4, p. 100023,2021 . [Online]. Available: https ://www. sciencedirect.com/science/article/pii / S2666827021000049.

[57] M. Irfan, L. Marcenaro, and L. Tokarchuk, "Crowd analysis using visual and non-visual sensors, a survey," in 2016 IEEE Global Conference on Signal and Information Processing (GlobalSIP), IEEE, 2016, pp. 1249-1254.
[58] J. C. Silveira Jacques Junior, S. R. Musse, and C. R. Jung, "Crowd analysis using computer vision techniques," IEEE Signal Processing Magazine, vol. 27, no. 5, pp. 66-77, 2010.

[59] T. Li, H. Chang, M. Wang, B. Ni, R. Hong, and S. Yan, "Crowded scene analysis: A survey," IEEE Transactions on Circuits and Systems for Video Technology, vol. 25, no. 3, pp. 367-386, 2015.

[60] S. T. Kouyoumdjieva, P. Danielis, and G. Karlsson, "Survey of non-image-based approaches for counting people," IEEE Communications Surveys Tutorials, vol. 22, no. 2, pp. 1305-1336, 2020.

[61] R. K. Ganti, F. Ye, and H. Lei, "Mobile crowdsensing: Current state and future challenges," IEEE Communications Magazine, vol. 49, no. 11, pp. 32-39, 2011.

[62] N. D. Lane, E. Miluzzo, H. Lu, D. Peebles, T. Choudhury, and A. T. Campbell, "A survey of mobile phone sensing," IEEE Communications Magazine, vol. 48, no. 9, pp. 140-150, 2010.

[63] J. A. Burke et al., "Participatory sensing," in Proceedings of the World Sensor Web Workshop, in Conjunction with ACM SenSys 2006, Boulder, CO, Oct. 2006.

[64] A. Olivo, G. Maternini, and B. Barabino, "Empirical Study on the Accuracy and Precision of Automatic Passenger Counting in European Bus Services," The Open Transportation Journal, vol. 13, pp. 250-260, 2019.

[65] C.-H. Chen, Y.-C. Chang, T.-Y. Chen, and D.-J. Wang, "People counting system for getting in/out of a bus based on video processing," in 2008 Eighth International Conference on Intelligent Systems Design and Applications, vol. 3, 2008, pp. 565-569.

[66] J. W. Choi, X. Quan, and S. H. Cho, "Bi-directional passing people counting system based on IR-UWB radar sensors," IEEE Internet of Things Journal, vol. 5, no. 2, pp. 512-522, 2018.

[67] S. Vidyasagaran, S. R. Devi, A. Varma, A. Rajesh, and H. Charan, "A low cost IoT based crowd management system for public transport," in 2017 International Conference on Inventive Computing and Informatics (ICICI), 2017, pp. 222-225.

[68] B. F. Nielsen, L. Frølich, O. A. Nielsen, and D. Filges, "Estimating passenger numbers in trains using existing weighing capabilities," Transportmetrica A: Transport Science, vol. 10, no. 6, pp. 502-517, 2014. [Online]. Available: https://doi.org/10.1080/23249935.2013. 795199.

[69] A. J. Kotz, D. B. Kittelson, and W. F. Northrop, "Novel vehicle mass-based automated passenger counter for transit applications," Transportation Research Record, vol. 2563, no. 1, pp. 37-43, 2016. [Online]. Available: https://doi.org/10.3141/2536-05.

[70] D.-Y. Chen and P.-C. Huang, "Visual-based human crowds behavior analysis based on graph modeling and matching," IEEE Sensors Journal, vol. 13, no. 6, pp. 2129-2138, 2013.

[71] P. Chato, D. J. M. Chipantasi, N. Velasco, S. Rea, V. Hallo, and P. Constante, "Image processing and 
artificial neural network for counting people inside public transport," in 2018 IEEE Third Ecuador Technical Chapters Meeting (ETCM), 2018, pp. 1-5.

[72] J. Zhang, J. Liu, and Z. Wang, "Convolutional neural network for crowd counting on metro platforms," Symmetry, vol. 13, no. 4, 2021. [Online]. Available: https://www.mdpi.com/2073-8994/13/4/703.

[73] M. Abdou and A. Erradi, "Crowd counting: A survey of machine learning approaches," in 2020 IEEE International Conference on Informatics, IoT, and Enabling Technologies (ICIoT), 2020, pp. 48-54.

[74] H. Yu, Z. He, and J. Liu, "A vision-based method to estimate passenger flow in bus," in 2007 International Symposium on Intelligent Signal Processing and Communication Systems, 2007, pp. 654-657.

[75] T. Yang, Y. Zhang, D. Shao, and Y. Li, "Clustering method for counting passengers getting in a bus with single camera," Optical Engineering, vol. 49, no. 3, pp. 1-10, 2010. [Online]. Available: https://doi.org/ 10.1117/1.3374439.

[76] S. Sun, N. Akhtar, H. Song, C. Zhang, J. Li, and A. Mian, "Benchmark data and method for real-time people counting in cluttered scenes using depth sensors," IEEE Transactions on Intelligent Transportation Systems, vol. 20, no. 10, pp. 3599-3612, 2019.

[77] G. Liu, Z. Yin, Y. Jia, and Y. Xie, "Passenger flow estimation based on convolutional neural network in public transportation system," Knowledge-Based Systems, vol. 123, pp. 102-115, 2017. [Online]. Available: https ://www. sciencedirect.com/science/article/pii/ S0950705117300849.

[78] A. V. Meghana, V. Sarode, D. Tambade, A. Marathe, and N. Charniya, "Automated crowd management in bus transport service," in 2020 International Conference on Electronics and Sustainable Communication Systems (ICESC), 2020, pp. 104-109.

[79] X. Ding, Z. Lin, F. He, Y. Wang, and Y. Huang, "A deeply-recursive convolutional network for crowd counting," in 2018 IEEE International Conference on Acoustics, Speech and Signal Processing (ICASSP), 2018, pp. 1942-1946.

[80] S.-Y. Cho, T. W. S. Chow, and C.-T. Leung, "A neuralbased crowd estimation by hybrid global learning algorithm," IEEE Transactions on Systems, Man, and Cybernetics, Part B (Cybernetics), vol. 29, no. 4, pp. 535-541, 1999.

[81] G. García-Bunster and M. Torres-Torriti, "A densitybased approach for effective pedestrian counting at bus stops," in 2009 IEEE International Conference on Systems, Man and Cybernetics, 2009, pp. 3434-3439.

[82] G. Garcia-Bunster, M. Torres-Torriti, and C. Oberli, "Crowded pedestrian counting at bus stops from perspective transformations of foreground areas," IET Computer Vision, vol. 6, no. 4, pp. 296-305, 2012.

[83] M. Andersson, J. Rydell, and J. Ahlberg, "Estimation of crowd behavior using sensor networks and sensor fusion," in 2009 12th International Conference on Information Fusion, 2009, pp. 396-403.
[84] W.-C. Lin, W. K. G. Seah, and W. Li, "Exploiting radio irregularity in the Internet of Things for automated people counting," in 2011 IEEE 22nd International Symposium on Personal, Indoor and Mobile Radio Communications, 2011, pp. 1015-1019.

[85] T. Tikkanen, "People detection and tracking using a network of low-cost depth cameras," Ph.D. dissertation, Jan. 2014.

[86] A. Tyndall, R. Cardell-Oliver, and A. Keating, "Occupancy estimation using a low-pixel count thermal imager," IEEE Sensors Journal, vol. 16, no. 10, pp. 37843791, 2016.

[87] I. Amin, A. J. Taylor, F. Junejo, A. Al-Habaibeh, and R. Parkin, "Automated people-counting by using lowresolution infrared and visual cameras," Measurement, vol. 41, pp. 589-599, Jul. 2008.

[88] S. Yaseen, A. Al-Habaibeh, D. Su, and F. Otham, "Real-time crowd density mapping using a novel sensory fusion model of infrared and visual systems," Safety Science, vol. 57, pp. 313-325, 2013.

[89] A. Gomez, F. Conti, and L. Benini, "Thermal imagebased CNN's for ultra-low power people recognition," in Proceedings of the 15th ACM International Conference on Computing Frontiers, ser. CF '18, Ischia, Italy: Association for Computing Machinery, 2018, pp. 326-331. [Online]. Available: https://doi.org/10. 1145/3203217.3204465.

[90] Y. Jiang, Y. Miao, B. Alzahrani, A. Barnawi, R. Alotaibi, and L. Hu, "Ultra large-scale crowd monitoring system architecture and design issues," IEEE Internet of Things Journal, vol. 8, no. 13, pp. $10356-$ 10366, 2021.

[91] A. Lesani, E. Nateghinia, and L. F. Miranda-Moreno, "Development and evaluation of a real-time pedestrian counting system for high-volume conditions based on 2D LiDAR," Transportation Research Part C: Emerging Technologies, vol. 114, pp. 20-35, 2020.

[92] M. Hashimoto, A. Tsuji, A. Nishio, and K. Takahashi, "Laser-based tracking of groups of people with sudden changes in motion," in 2015 IEEE International Conference on Industrial Technology (ICIT), 2015, pp. 315-320.

[93] S.-i. Akamatsu, N. Shimaji, and T. Tomizawa, "Development of a person counting system using a $3 \mathrm{D}$ laser scanner," in 2014 IEEE International Conference on Robotics and Biomimetics (ROBIO 2014), 2014, pp. 1983-1988.

[94] P. G. Kannan, S. P. Venkatagiri, M. C. Chan, A. L. Ananda, and L.-S. Peh, "Low cost crowd counting using audio tones," in Proceedings of the 10th ACM Conference on Embedded Network Sensor Systems, Toronto, Ontario, Canada: Association for Computing Machinery, 2012, pp. 155-168. [Online]. Available: https://doi.org/10.1145/2426656.2426673.

[95] R. Agarwal, S. Kumar, and R. M. Hegde, "Algorithms for crowd surveillance using passive acoustic sensors over a multimodal sensor network," IEEE Sensors Journal, vol. 15, no. 3, pp. 1920-1930, 2015. 
[96] M. A. Al Hafiz Khan, H. M. S. Hossain, and N. Roy, "SensePresence: infrastructure-less occupancy detection for opportunistic sensing applications," in 2015 16th IEEE International Conference on Mobile Data Management, vol. 2, 2015, pp. 56-61.

[97] O. Shih and A. Rowe, "Occupancy estimation using ultrasonic chirps," in Proceedings of the ACM/IEEE Sixth International Conference on Cyber-Physical Systems, ser. ICCPS '15, Seattle, Washington: Association for Computing Machinery, 2015, pp. 149-158. [Online]. Available: https://doi .org/10.1145/2735960. 2735969.

[98] S. Di Domenico, G. Pecoraro, E. Cianca, and M. De Sanctis, "Trained-once device-free crowd counting and occupancy estimation using WiFi: A Doppler spectrum based approach," in 2016 IEEE 12th International Conference on Wireless and Mobile Computing, Networking and Communications (WiMob), 2016, pp. 1-8.

[99] S. Bartoletti, A. Conti, and M. Z. Win, "Devicefree counting via wideband signals," IEEE Journal on Selected Areas in Communications, vol. 35, no. 5, pp. 1163-1174, 2017.

[100] R. F. Brena, E. Escudero, C. Vargas-Rosales, C. E. Galvan-Tejada, and D. Munoz, "Device-Free Crowd Counting Using Multi-Link Wi-Fi CSI Descriptors in Doppler Spectrum," Electronics, vol. 10, no. 3, 2021. [Online]. Available: https://www. mdpi .com/2079 9292/10/3/315.

[101] J.-H. Choi, J.-E. Kim, and K.-T. Kim, "People counting using IR-UWB radar sensor in a wide area," IEEE Internet of Things Journal, vol. 8, no. 7, pp. 58065821, 2021.

[102] Y. Yuan, J. Zhao, C. Qiu, and W. Xi, "Estimating crowd density in an RF-based dynamic environment," IEEE Sensors Journal, vol. 13, no. 10, pp. 3837-3845, 2013.

[103] S. Depatla, A. Muralidharan, and Y. Mostofi, "Occupancy Estimation Using Only WiFi Power Measurements," IEEE Journal on Selected Areas in Communications, vol. 33, no. 7, pp. 1381-1393, 2015.

[104] O. T. Ibrahim, W. Gomaa, and M. Youssef, "CrossCount: A Deep Learning System for Device-Free Human Counting Using WiFi," IEEE Sensors Journal, vol. 19, no. 21, pp. 9921-9928, 2019.

[105] W. Xi et al., "Electronic frog eye: Counting crowd using WiFi," in IEEE INFOCOM 2014 - IEEE Conference on Computer Communications, 2014, pp. 361369.

[106] M. De Sanctis, T. Rossi, S. Di Domenico, E. Cianca, G. Ligresti, and M. Ruggieri, "LTE signals for devicefree crowd density estimation through CSI secant set and SVD," IEEE Access, vol. 7, pp. 159943-159951, 2019.

[107] O. Oshiga, H. U. Suleiman, S. Thomas, P. Nzerem, L. Farouk, and S. Adeshina, "Human Detection For Crowd Count Estimation Using CSI of WiFi Signals," in 2019 15th International Conference on Electronics, Computer and Computation (ICECCO), 2019, pp. 1-6.
[108] F. Brockmann, M. Handte, and P. J. Marrón, "CutiQueue: people counting in waiting lines using Bluetooth low energy based passive presence detection," in 2018 14th International Conference on Intelligent Environments (IE), 2018, pp. 1-8.

[109] I. Sobron, J. Del Ser, I. Eizmendi, and M. Vélez, "Device-free people counting in IoT environments: New insights, results, and open challenges," IEEE Internet of Things Journal, vol. 5, no. 6, pp. 4396-4408, 2018.

[110] A. Capozzoli et al., "SAR tomography with optimized constellation and its application to forested scenes," Fondazione Giorgio Ronchi, vol. LXV, no. 3, pp. 367375, 2010.

[111] A. Capozzoli, C. Curcio, I. Iudice, A. Liseno, and S. Savarese, "Fast imaging of vegetation on GPUs based on Non-Uniform FFTs," in 2011 International Conference on Electromagnetics in Advanced Applications, 2011, pp. 1205-1208.

[112] C. Oberli, M. Torres-Torriti, and D. Landau, "Performance evaluation of UHF RFID technologies for realtime passenger recognition in intelligent public transportation systems," IEEE Transactions on Intelligent Transportation Systems, vol. 11, no. 3, pp. 748-753, 2010.

[113] R. Want, "Near field communication," IEEE Pervasive Computing, vol. 10, no. 3, pp. 4-7, 2011.

[114] U. Demir Alan and D. Birant, "Server-based intelligent public transportation system with NFC," IEEE Intelligent Transportation Systems Magazine, vol. 10, no. 1, pp. 30-46, 2018.

[115] J. Weppner, P. Lukowicz, U. Blanke, and G. Tröster, "Participatory Bluetooth scans serving as urban crowd probes," IEEE Sensors Journal, vol. 14, no. 12, pp. 4196-4206, 2014.

[116] V. Kostakos, T. Camacho, and C. Mantero, "Wireless detection of end-to-end passenger trips on public transport buses," in 13th International IEEE Conference on Intelligent Transportation Systems, 2010, pp. 17951800.

[117] A. Basalamah, "Sensing the crowds using Bluetooth Low Energy tags," IEEE Access, vol. 4, pp. 42254233, 2016.

[118] "Immuni documentation," Tech. Rep. [Online]. Available: https://www.immuni.italia.it/.

[119] M. Nitti, F. Pinna, L. Pintor, V. Pilloni, and B. Barabino, "iABACUS: A Wi-Fi-based automatic bus passenger counting system," Energies, vol. 13, no. 6, 2020. [Online]. Available: https://www.mdpi.com/ 1996-1073/13/6/1446.

[120] K. Shibata and H. Yamamoto, "People crowd density estimation system using deep learning for radio wave sensing of cellular communication," in 2019 International Conference on Artificial Intelligence in Information and Communication (ICAIIC), 2019, pp. 143-148.

[121] J. A. del Peral-Rosado, R. Raulefs, J. A. LópezSalcedo, and G. Seco-Granados, "Survey of cellular mobile radio localization methods: From $1 \mathrm{G}$ to 
5G," IEEE Communications Surveys Tutorials, vol. 20, no. 2, pp. 1124-1148, 2018.

[122] M. G. Demissie, S. Phithakkitnukoon, T. Sukhvibul, F. Antunes, R. Gomes, and C. Bento, "Inferring passenger travel demand to improve urban mobility in developing countries using cell phone data: A case study of Senegal," IEEE Transactions on Intelligent Transportation Systems, vol. 17, no. 9, pp. 2466-2478, 2016. 